\title{
HaMiPla Best Paper Sitzungen - Ihre Favoriten 2014
}

\author{
HaMiPla Best Paper Award - Your Favourites in 2014
}

K. Maier, K. J. Prommersberger, R. E. Giunta

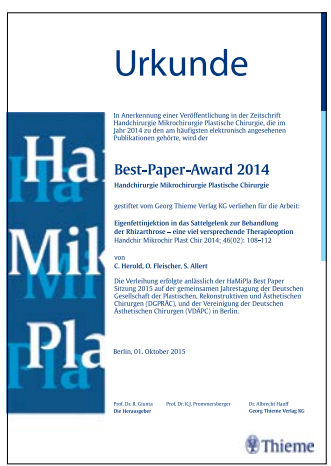

Nach dem großen Erfolg im vergangenen Jahr, in dem wir erstmalig den HaMiPla Best-PaperAward verliehen haben, konnten wir 2015 gleich auf 2 Jahrestagungen - einmal in Ludwigsburg im Rahmen des DGH-Kongresses und einmal in Berlin im Rahmen der DGPRÄC und VDÄPCJahrestagung - die HaMipla-Best-Paper-Awards 2014 verleihen. 10 Arbeiten wurden mit diesem Award ausgezeichnet, der mit einem Buchgutschein und einer Urkunde verbunden ist: Jeweils 5 Arbeiten hatten einen handchirurgischem Schwerpunkt und 5 Veröffentlichungen waren mit plastisch-chirurgischem Hintergrund. Zugrundeliegendes Auswahlkriterium war die Anzahl der Volltextdownloads im vergangenen Jahr.

Gemeinsam mit den Herausgebern Prof. Riccardo Giunta und Prof. Karl-Josef Prommersberger sowie den jeweiligen Kongresspräsidenten Prof. Max Haerle für die DGH und Dr. Uwe von Fritschen sowie Dr. Bernd Hartmann für die DGPRÄC wurde für beide Kongresse jeweils eine HaMiPlaBest-Paper Award Session konzipiert und in das Kongressprogramm aufgenommen. Im Rahmen dieser Sessions hatten die Autoren Gelegenheit ihre Publikationen und den Hintergrund ihrer Studien und ihrer Forschung einem interessierten Publikum zu präsentieren. Prof. Giunta und Prof. Prommersberger moderierten kurzweilig durch die vielfältigen Präsentationen.

Auf der DGH-Tagung (Donnerstag, 24.9.2015) lag der Fokus der Best-Paper-Präsentationen auf den Themen Infektionen und Arthroskopie ( $\bullet$ Abb. 1): D. Schmauss präsentierte die Ergebnisse zur Fragestellung: Primär adaptierende vs. sekundäre Wundverschlüsse bei Infektionen der Hand - Unterschiede und Vorteile [1]. Eine viel diskutierte Fragestellung, wie auch die anschließenden Rückmeldungen aus dem Publikum zeigten.
Thematisch dazu passend präsentierte F. Stang die Daten einer Evaluation zum zweizeitigen Therapieregimes bei Handinfektionen [2]. Dabei ging er auch der Frage nach, ob eine akute Handinfektionen immer ein Oberarzteingriff sein sollte. Das Publikum - nach der ersten Präsentation schon eingestimmt, diskutierte auch hier intensiv über eigene Erfahrungen und Einschätzungen. Nicht umsonst war dies eines der am häufigsten gelesenen Papers.

Der zweite Teil der Session griff das Thema Arthroskopie auf:

M. Lutz stellte die Ergebnisse vor zum Thema: Arthroskopisch assistierte Versorgung dorsal dislozierter, distaler, intraartikulärer Speichenfrakturen - Technik und Ergebnisse [3]. Eine viel beachtete Arbeit und ein vom Publikum sehr positiv aufgenommener Ansatz. Dass die Preisauszeichnung dazuhin an einen Kollegen aus dem benachbarten Ausland ging, wurde von allen Beteiligten sehr begrüßt, zeigt es doch die Zusammengehörigkeit der deutschsprachigen Handchirurgie. H.-G. Tünnerhoff präsentierte die Arbeit zur arthroskopischen Refixation des TFCC bei ulnarer Ruptur [4], die neben den operativen Ergebnissen auch durch die herausragenden Zeichnungen von M. Langer bestach. Den Abschluss übernahm G. Broccoli mit seinem Vortrag zur Darstellung der Grenzen der arthroskopischen Behandlung degenerativer Läsionen des ulnokarpalen Komplexes in Abhängigkeit der Ulnavarianz [5]. Auch hier wurde viel diskutiert, so dass die Moderatoren durchgängig gefordert waren, die vorgegebenen Zeiten einzuhalten.

Nach der „Premiere“ in Ludwigsburg ging die zweite Runde unserer HaMiPla-Best-PaperAward-Verleihung in Berlin im Rahmen der DGPRÄC-Jahrestagung ebenfalls sehr erfolgreich über die Bühne. Hier fanden sich Referenten und interessiertes Publikum am Donners-

\section{Bibliografie}

DOI http://dx.doi.org/ 10.1055/s-0035-1565126 Handchir Mikrochir Plast Chir 2015; 47: 408-409 (c) Georg Thieme Verlag KG Stuttgart · New York ISSN 0722-1819

Korrespondenzadresse Katrin Maier Programmplanerin Georg Thieme Verlag KG Klinik und Praxis Ruedigerstraße 14 70469 Stuttgart katrin.maier@thieme.de

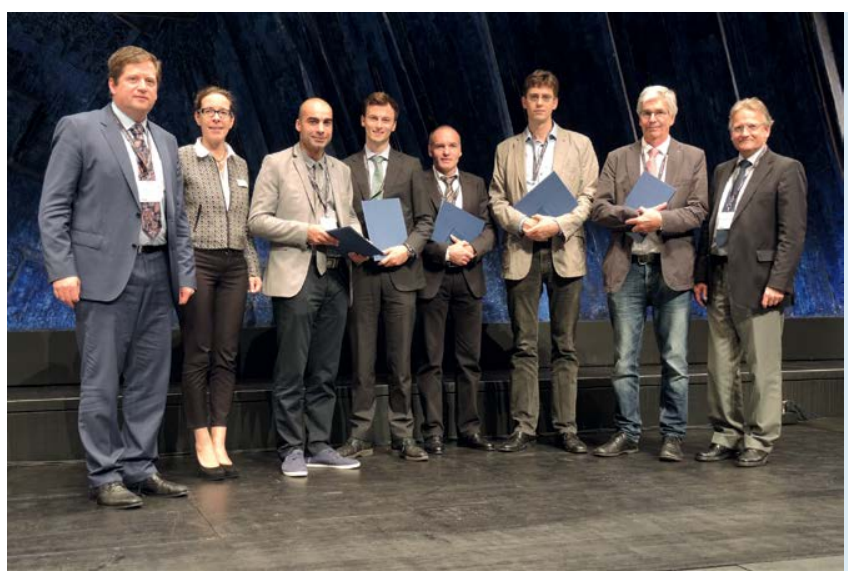

Abb. 1 Preisträger und Referenten der HaMiPla-Best-Paper-Award-Session auf dem DGH-Kongress 2015. Von links nach rechts: R. Giunta (Herausgeber HaMiPla), K. Maier (Thieme Verlag), G. Broccoli, D. Schmauss, M. Lutz, F. Stang, H.-G. Tünnerhoff, K.-J. Prommersberger (Herausgeber HaMiPla). 


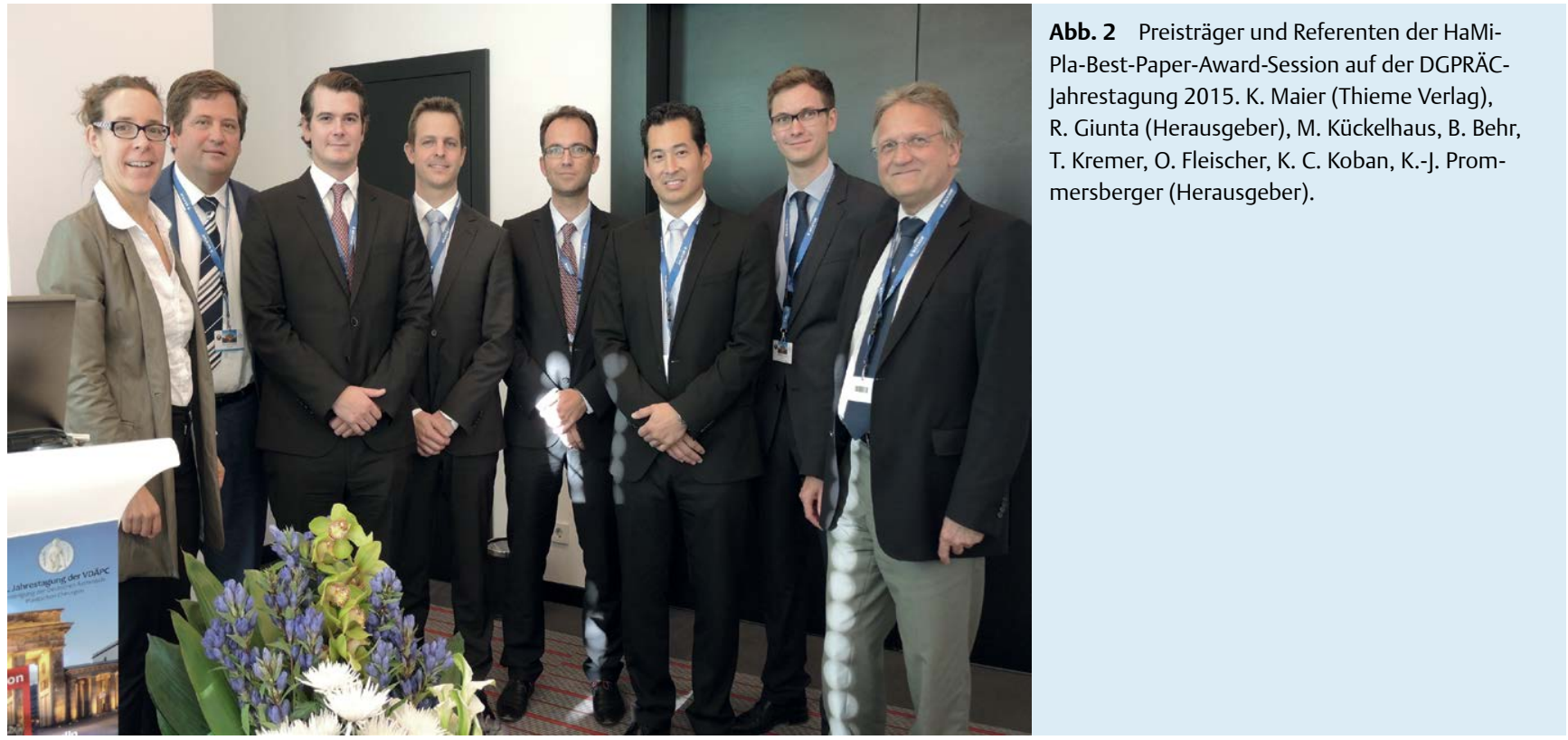

tag, den 1.10.2015 zur gemeinsamen Sitzung zusammen (० Abb. 2).

Den Auftakt machte O. Fleischer mit einem Vortrag zum Thema: Eigenfettinjektion in das Sattelgelenk zur Behandlung der Rhizarthrose - eine vielversprechende Therapieoption [6]. Er berichtete über die Ergebnisse dieser Methode. Ein wichtiges Thema mit hohem Alltagsbezug, das im Anschluss unter vielfältigen Aspekten diskutiert wurde. Anschließend folgten 3 Vorträge aus Bochum (und Ludwigshafen): die Kollegen aus der BG Klinik Bergmannsheil um Herrn Prof. Marcus Lehnhardt sahnten dieses Jahr gleich dreifach ab. B. Behr präsentierte die Ergebnisse der Arbeitsgruppe die sich mit den Therapieoptionen zur Rekonstruktion der dorsalen Rumpfwand beschäftigten [7]. Ein Thema, das alle immer wieder betrifft und entsprechend interessant war der anschließende Austausch im Publikum. M. Kückelhaus berichtete von den Fortschritten in der Gesichtstransplantation [8]. Eine Arbeit, die zum Teil in Boston entstand. Kein alltägliches Thema, das viele Fragezeichen aufwirft, da neben den klassisch chirurgischen Themen, viele ethischen Aspekte und psychologische Fragen diskutiert werden. Den Abschluss des Bochumer Trios machte T. Kremer, als Vertreter aus der BGU Ludwigshafen, mit seiner Präsentation zur mikrochirurgischen Ausbildung in Deutschland - Ergebnisse einer Umfrage unter Weiterbildungsassistenten und Weiterbildern [9]: auch hier interessante Ergebnisse, die vom Publikum gern aufgenommen wurden. K. C. Koban stellte abschließend neue Methoden zur 3D-Bilderfassung vor: 3D Bilderfassung und Analyse in der Plastischen Chirurgie mit Smartphone und Tablet: eine Alternative zu professionellen Systemen? [10] Die Frage konnte nicht abschließend geklärt werden, aber die Diskussion zeigte, wohin sich das Ganze entwickeln kann.

Die 10 Artikel können über folgenden Link abgerufen werden:

http://tinyurl.com/nck6zwa

\section{Literatur}

1 Schmauss D, Finck T, Lohmeyer JA et al. Primär adaptierende vs. sekundäre Wundverschlüsse bei Infektionen der Hand - Unterschiede und Vorteile. Handchir Mikrochir Plast Chir 2014; 46: 56-60

2 Bergmann PA, Mauss KL, Liodaki ME et al. Handinfektionen: Evaluation eines zweizeitigen Therapieregimes. Handchir Mikrochir Plast Chir 2014; 46: 49-55

3 Lutz M, Wieland T, Deml C et al. Arthroskopisch assistierte Versorgung dorsal dislozierter distaler intraartikulärer Speichenfrakturen - Technik und Ergebnisse. Handchir Mikrochir Plast Chir 2014; 46: 271-277

4 Tünnerhoff $H-G$, Langer $M$. Arthroskopische Refixation des TFCC bei ulnarer Ruptu. Handchir Mikrochir Plast Chir 2014; 46: 286-294

5 Broccoli G, Vaske B, Müller L et al. Grenzen der arthroskopischen Behandlung degenerativer Läsionen des ulnokarpalen Komplexes in Abhängigkeit der Ulnavarianz. Handchir Mikrochir Plast Chir 2014; 46: $151-162$

6 Herold C, Fleischer O, Allert S. Eigenfettinjektion in das Sattelgelenk zur Behandlung der Rhizarthrose - eine viel versprechende Therapieoption. Handchir Mikrochir Plast Chir 2014; 46: 108-112

7 Behr B, Hirsch T, Goertz $O$ et al. Therapieoptionen zur Rekonstruktionen der dorsalen Rumpfwand. Handchir Mikrochir Plast Chir 2014; 46: 90-96

8 Kueckelhaus $M$, Lehnhardt $M$, Fischer $S$ et al. Fortschritte in der Gesichtstransplantation. Handchir Mikrochir Plast Chir 2014; 46: 206-213

9 Kolbenschlag J, Gehl B, Daigeler A et al. Mikrochirurgische Ausbildung in Deutschland - Ergebnisse einer Umfrage unter Weiterbildungsassistenten und Weiterbildern. Handchir Mikrochir Plast Chir 2014; 46: $234-241$

10 Koban KC, Leitsch S, Holzbach T et al. 3D Bilderfassung und Analyse in der Plastischen Chirurgie mit Smartphone und Tablet: eine Alternative zu professionellen Systemen? Handchir Mikrochir Plast Chir 2014; 46: 97-104 